\title{
The Quantum Equivalent in Photo-electric Conduction.
}

I F light of frequency $\nu$ is sent through a cold gas which does not absorb it, sensitised by admixture of a second gas which can absorb the light; and the pressure is such that the mean time between two collisions is of the same order of magnitude as the mean life of the excited state of the gas; all those spectral lines of the non-absorbing gas appear which have a smaller excitation energy than $h \nu$. On the other hand, those which require more energy than this are not seen. Results with mixtures of mercury and thorium vapours, and of mercury and silver vapours, using the light of the $2536.7 \AA \mathrm{Hg}$ line, agree, on the whole, closely with the above statement. The method can also be used in fixing the series relations between the lines of an element, since it allows us to determine which spectral lines can be excited by an amount of energy smaller than a given amount. Results have been obtained at Göttingen for lead and bismuth, which will shortly be published.

In a paper in the Zeitschrift für Physik, I7-3, August 23, p. 202, Messrs. G. Carlo and J. Franck consider the theory more closely, and describe experiments which agree with their conclusions. The sensitising gas $A$ has, as the longest wave of its absorption series, a line of frequency $\nu$, while the corresponding line of the fluorescing gas has the frequency $\nu_{1}$. Suppose $h \nu: h \nu_{1}:: \mathrm{I}: \frac{3}{4} ;$ then if light of frequency $\nu$ is used, both $\nu$ and $\nu_{1}$ will appear. The elementary act of transmission of energy from an excited atom of $\mathrm{A}$ to an atom of $\mathrm{B}$ will take place in such a way that $\frac{1}{4} h \nu$ is converted into energy of translation of the colliding atoms. If the temperature is so low that the kinetic energy of temperature movement is small, compared with $h v$, the atom receives, besides its excitation energy, the kinetic energy $\frac{1}{2} m_{1} v_{1}^{2}=\frac{h v}{4} \times \frac{\mathrm{I}}{\mathrm{I}+m_{1} / m} \cdot$ This abnormal velocity of the excited atom of $\mathrm{B}$ produces a Doppler effect; the effective frequency is $\nu_{2}=v_{1}\left(\mathrm{I}+\cos \phi v_{1} / c\right)$, and this is not absorbed by the other practically resting atoms of $\mathrm{B}$.

An experiment with sodium vapour, sensitised with pure argon, has verified the theory. Sodium vapour can be made to fluoresce by means of the zinc line, $3303 \AA$, and the sodium can also be excited directly by the $\mathrm{D}$ line. Conditions were so adjusted that the tube appeared equally bright with either of these sources, and the light from the fluorescing sodium, in each case, was passed through an absorption tube, containing sodium at a suitable temperature. The light excited by the $D$ line was completely extinguished, while that obtained with the zinc line was much less weakened.

If, in a second experiment, light of frequency $\nu_{1}$ passes through the mixture of gases $A$ and $B$, an emission of $\nu$ from $A$ can result only if the temperature of the gas is so high that the energy difference $h \nu / 4$, which is lacking for the excitation of $A$, can be obtained on collision from the kinetic energy of the atoms. Experiments have been made with thallium and mercury, and with cadmium and mercury, using the $\mathrm{Hg}$ line $2536.7 \AA$. The quartz vessel containing the vapours was placed in an electric oven, which could be raised to $800^{\circ} \mathrm{C}$.; and strong fluorescence was obtained with thallium. In this case it was possible that a thallium line, the excitation energy of which amounts to 5.5 volts, while the energy of $2536.7 \AA$ corresponds to 4.9 volts only, was due to a double or step-by-step process of excitation. At $800^{\circ} \mathrm{C}$. part of the atoms are no longer in the normal state; and apparently there is a I volt excitation step, from which, up to the 5.5 volts stage, only 4.5 volts would be required, or more than for $2536.7 \AA$.

With cadmium and mercury this difficulty does not arise, and the confirmation of the theory is direct. It is possible, however, to draw conclusions from the relative intensity of the lines in the fluorescent spectrum of thallium, as compared with the ordinary spectrum of this substance. The differences are ascribed to the differences in absorption, due to the presence or absence of the Doppler effect previously described; and the combined results of the two experiments seem to prove, conclusively, that quantum energy and translation energy can work together, as an elementary act, to produce excitation of the atom.

\section{Early Methods of Oil Painting. ${ }^{1}$}

\section{By Prof. A. P. LAURIE.}

I $T$ is evident both from the manuscript of Theophilus and the manuscript of Eraclius that the properties of such drying oils as linseed oil and walnut oil were thoroughly understood as early as the I2th century, if not earlier.

The methods used in their preparation differ very little from the best practice of to-day. The refining and bleaching of the oil and the use of driers was well understood, nor is there any indication, in passing from those earlier recipes to those of the $I_{5}$ th century, that any new discovery of importance was made at the time of the brothers Van Eyck. Passing to later times, Vasari directs that pigments are to be ground in walnut oil or linseed oil, and this is all that is necessary; he recommends the use of walnut oil as less liable to darken with time.

Many recipes for varnishes are given, and, as neither spirit of turpentine nor alcohol was available in commercial quantities until the end of the I5th century, these varnishes are what we should now describe as oil varnishes, consisting of resins dissolved in hot oil. The natural balsams of the pine, resin, mastic, and sandarac, often all mixed together, were $h^{1}$ Synopsis of lecture delivered at the Royal Academy of Arts, London, on Wernesday, November 14 .

$$
\text { NO. } 2824 \text {, VOL I I 2] }
$$

used in the preparation of these varnishes. The proportion of resinous material to oil being very high, the varnishes were consequently very sticky and had to be heated and rubbed on with the hand. Spirit varnishes corresponding to the mastic varnishes of to-day are found in 16 th century and later recipes.

The evidence of the accounts preserved at Ely and Westminster show that both oil and varnish were used in painting on walls during the I 3 th and I4th centuries, this being the northern tradition, while the Italian tradition was the use of egg as a medium. There is no indication in these recipes of any special secret differing from what we know to-day.

These early pictures were painted on a wood panel sometimes covered with strips of linen and coated with a gesso made of parchment size and whitening or plaster of Paris which had been soaked in water until it lost its binding properties. Recent experiments carried out by Mr. Thompson at the Heriot-Watt College, Edinburgh, on an old I6th century panel have revealed the fact that this panel was coated with a non-absorbent gesso upon which a very thin layer of absorbent gesso was laid so as to ensure the binding of the oil to the surface of the gesso and, at the same time, to preserve from staining the pure white surface 